\title{
Synthesis and biological evaluation of lycorine derivatives as dual inhibitors of human acetylcholinesterase and butyrylcholinesterase
}

\author{
Yue-Hu Wang ${ }^{1 *}$, Qin-Li Wan ${ }^{2}$, Cheng-Ding Gu ${ }^{3}$, Huai-Rong Luo ${ }^{2}$ and Chun-Lin Long ${ }^{1,4^{*}}$
}

\begin{abstract}
Background: Alzheimer's disease (AD) is a neurologically degenerative disorder that affects more than 20 million people worldwide. The selective butyrylcholinesterase (BChE) inhibitors and bivalent cholinesterase (ChE) inhibitors represent new treatments for $A D$.

Findings: A series of lycorine derivatives (1-10) were synthesized and evaluated for anti-cholinesterase activity. Result showed that the novel compound 2-O-tert-butyldimethylsilyl-1-O-(methylthio)methyllycorine (7) was a dual inhibitor of human acetylcholinesterase (hAChE) and butyrylcholinesterase (hBChE) with $I_{50}$ values of $11.40 \pm 0.66 \mu \mathrm{M}$ and $4.17 \pm 0.29 \mu \mathrm{M}$, respectively. The structure-activity relationships indicated that (i) the 1-O-(methylthio)methyl substituent in lycorine was better than the 1-O-acetyl group for the inhibition of cholinesterase; (ii) the acylated or etherified derivatives of lycorine and lycorin-2-one were more potent against hBChE than hAChE; and (iii) the oxidation of lycorine at C-2 decreases the activity.
\end{abstract}

Conclusion: Acylated or etherified derivatives of lycorine are potential dual inhibitors of hBChE and hAChE. Hence, further study on the modification of lycorine for ChE inhibition is necessary.

Keywords: Amaryllidaceae alkaloids, Lycorine, Acetylcholinesterase, Butyrylcholinesterase

\section{Findings}

Alzheimer's disease (AD) is a neurologically degenerative disorder that affects more than 20 million people worldwide [1], and is the third-most costly disease after cardiovascular disease and cancer [2]. The neuropathological hallmarks of the disease include $\beta$-amyloid $(A \beta)$ plaques, neurofibrillary tangles, and synaptic loss. Based on the cholinergic hypothesis, the symptoms of $\mathrm{AD}$ are the result of the reduction in brain acetylcholine $(\mathrm{ACh})$ activity due to the catabolism of ACh by its principal hydrolytic enzyme acetylcholinesterase (AChE). AChE inhibition is the current approach for $\mathrm{AD}$ treatment. Tacrine, donepezil, rivastigmine, and galanthamine are all examples of typical AChE inhibitory drugs [3].

\footnotetext{
* Correspondence: wangyuehu@mail.kib.ac.cn; chunlinlong@hotmail.com ${ }^{1}$ Key Laboratory of Economic Plants and Biotechnology, Kunming Institute of Botany, Chinese Academy of Sciences, Kunming 650201, PR China ${ }^{4}$ College of Life and Environmental Sciences, Minzu University of China, Beijing 100081, PR China

Full list of author information is available at the end of the article
}

Similar to AChE, butyrylcholinesterase (BChE) can also inactivate ACh. The reduction in ACh is usually accompanied by a decrease in AChE activity. By contrast, BChE in $\mathrm{AD}$ remains at normal levels or even elevated in the brain. BChE may be a significant contributor to the observed loss of ACh in AD [4]. Furthermore, BChE inhibition can lower $\mathrm{A} \beta$ peptide $[5,6]$. $\mathrm{BChE}$ is essential in $\mathrm{AD}$ plaque maturation [7]. Selective $\mathrm{BChE}$ inhibition may be crucial in the mid to late stages of $\mathrm{AD}$ pathogenesis to circumvent further decline in mental and cognitive ability as the depletion of cholinergic neurons persists [3]. Hence, selective $\mathrm{BChE}$ inhibitors or bivalent $\mathrm{ChE}$ inhibitors represent a new treatment for AD.

Lycorine, the most frequent alkaloid in Amaryllidaceae plants, has very weak inhibitory activity against electric eel acetylcholinesterase (eeAChE), with an $\mathrm{IC}_{50}$ value of $213 \mu \mathrm{M}[8]$. Acylated or etherified derivatives of lycorine, such'as 1-O-acetyllycorine and 1-O-acetyl-2-O-tert-butyldimethylsilyllycorine (6, Figure 1), possess potent activity against eeAChE $[8,9]$. However, the inhibitory effect of analogues on $\mathrm{BChE}$ has not been reported. In our continuing
(C) Chemistry Central

(c) 2012 Wang et al.; licensee Chemistry Central Ltd. This is an Open Access article distributed under the terms of the Creative Commons Attribution License (http://creativecommons.org/licenses/by/2.0), which permits unrestricted use, distribution, and reproduction in any medium, provided the original work is properly cited. 


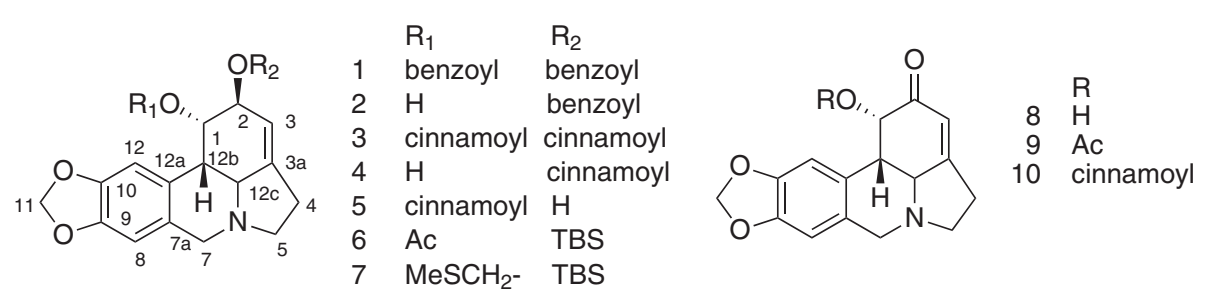

Figure 1 Chemical structures of compounds under study.

work on Amaryllidaceae alkaloids [10-12], the present study reports the synthesis of lycorine derivatives (1-10), and their biological evaluation for inhibition of $\mathrm{ChE}$.

Previous researchers considered that a hydrogen-bond acceptor at the C-1 of lycorine is necessary for AChE inhibitory activity, and a bulky, lipophilic substituent, such as the TBS group, at C-2 increases the activity $[9,13]$. Therefore, in the present study, benzoic acid or cinnamic acid were used to acylate the $1-\mathrm{OH}$ and/or $2-\mathrm{OH}$ of lycorine and its $\mathrm{C}-1$ or $\mathrm{C}-2$ oxidation derivatives. Mono- or di-acylated derivatives (1-5, and 10) of lycorine and lycorin-2-one (8) were obtained by Steglich esterification (DCC/DMAP). Lycorine oxidation using pyridinium chlorochromate (PCC) in DMF yielded $\mathbf{8}$, and the acetylated analogue (9) of the latter was obtained by the reaction of $\mathbf{8}$ with $\mathrm{Ac}_{2} \mathrm{O}$ /pyridine. The $\mathrm{DMSO} / \mathrm{Ac}_{2} \mathrm{O}$ system was used to oxidate $\mathrm{C}-1$ of lycorine, with the protection of $1-\mathrm{OH}$ by the TBS group. However, 1-O-acetyl and 1-O-(methylthio)methyl derivatives (6 and 7) were obtained instead of the desired C-1 oxidation product.

The anti-ChE activity of these prepared lycorine derivatives (1-10) was evaluated by in vitro ChE inhibition assay, modified from Ellman's method [14]. The results were expressed as $\mathrm{IC}_{50}$ values and summarized in Table 1.

2-O-tert-Butyldimethylsilyl-1-O-(methylthio)methyllycorine (7) showed dual inhibitory activity against both $\mathrm{hAChE}\left(\mathrm{IC}_{50}=11.40 \pm 0.66 \mu \mathrm{M}\right)$ and $\mathrm{hBChE}\left(\mathrm{IC}_{50}=\right.$ $4.17 \pm 0.29 \mu \mathrm{M})$. The inhibitory potency of 7 was approximately four-fold stronger than that of galanthamine $\left(\mathrm{IC}_{50}=18.30 \pm 0.14 \mu \mathrm{M}\right)$ on hBChE. Compounds 1, and 2-4 also showed good effects on hBChE, with $\mathrm{IC}_{50}$ values of less than $20 \mu \mathrm{M}$.

Table 1 shows that the acylated or etherified derivatives $(\mathbf{1}, 3-5,7,9$, and 10) of lycorine and lycorin-2-one are more potent against hBChE than hAChE. The hBChE inhibitory activity of 1-O-trans-cinnamoyllycorine $\left(5, \mathrm{IC}_{50}=12.13 \pm 0.77 \mu \mathrm{M}\right)$ is about two-fold better than that of 1-O-trans-cinnamoyllycorin-2-one $\left(10, \mathrm{IC}_{50}=\right.$ $20.91 \pm 0.13 \mu \mathrm{M})$. This result implied that lycorine oxidation at $\mathrm{C}-2$ may decrease the activity.

A previous study reported that 1-O-acetyl-2-O-tertbutyldimethylsilyllycorine (6) showed significant inhibitory activity against ACh biotransformation by eeAChE $\left(K_{\mathrm{i}}=0.34 \mu \mathrm{M}\right)$ [9]. However, in the current study, 1-O-acetyl-2-O-tert-butyldimethylsilyllycorine was

Table 1 Inhibitory effect of compounds 1-10 on human AChE and BChE

\begin{tabular}{|c|c|c|c|}
\hline \multirow[b]{2}{*}{ Lycorine analogue } & \multirow[b]{2}{*}{ No. } & \multicolumn{2}{|l|}{$\mathrm{IC}_{50}(\mu \mathrm{M})$} \\
\hline & & hAChE & hBChE \\
\hline 1,2-O,O'-Dibenzoyllycorine & 1 & $>50$ & $7.72 \pm 0.26$ \\
\hline 2-O-Benzoyllycorine & 2 & $>50$ & $>50$ \\
\hline 1,2-O,O'-Di-trans-cinnamoyllycorine & 3 & $46.76 \pm 0.95$ & $17.45 \pm 0.19$ \\
\hline 2-O-trans-Cinnamoyllycorine & 4 & $>50$ & $19.74 \pm 1.37$ \\
\hline 1-O-trans-Cinnamoyllycorine & 5 & $>50$ & $12.13 \pm 0.77$ \\
\hline 1-O-Acetyl-2-O-tert-butyldimethylsilyllycorine & 6 & $>50$ & $>50$ \\
\hline 2-O-tert-Butyldimethylsilyl-1-O-(methylthio)methyllycorine & 7 & $11.40 \pm 0.66$ & $4.17 \pm 0.29$ \\
\hline Lycorin-2-one & 8 & $>50$ & $>50$ \\
\hline 1-O-Acetyllycorin-2-one & 9 & $>50$ & $44.46 \pm 0.88$ \\
\hline 1-O-trans-Cinnamoyllycorin-2-one & 10 & $>50$ & $20.91 \pm 0.13$ \\
\hline Tacrine (positive control) & & $0.26 \pm 0.015$ & $0.02 \pm 0.00$ \\
\hline Galanthamine (positive control) & & $1.60 \pm 0.14$ & $18.30 \pm 0.14$ \\
\hline
\end{tabular}


inactive $\left(\mathrm{IC}_{50}>50 \mu \mathrm{M}\right)$ against both of hAChE and hBChE. 1-O-(Methylthio)methyl substituent at C-1 of lycorine significantly increased the inhibitory activity against both of hAChE and hBChE in 7 compared with that of 6. Compound 7 was an unexpected product; its formation mechanism can be explained by a Pummerer rearrangement (Scheme 1) [15].

The bioassay result of compound 7 compared with those of other tested compounds showed that a bulky, lipophilic substituent at $\mathrm{C}-1$ or $\mathrm{C}-2$ of lycorine is necessary for the human $\mathrm{ChE}$ inhibitory activity. In addition, the substituted group at $\mathrm{C}-1$ is important in the activity.

The positive control tacrine showed a significant inhibitory effect on both hAChE and hBChE. However, tacrine is currently rarely used because of its hepatotoxicity [16]. Based on the results in the present study, modification of lycorine for the inhibition of ChE, especially of $\mathrm{hBChE}$, is necessary.

\section{Experimental Chemistry}

NMR spectra were recorded on Bruker AM-400 and DRX-500 spectrometers with TMS as an internal standard. ESIMS data were measured on an APIQstar-Pulsar-1 instrument and HREIMS on a Waters Autospec Premier P776 mass spectrometer. Column chromatography was performed over silica gel G (80-100 and 300-400 mesh), silica gel $\mathrm{H}(10-40 \mu \mathrm{m})$, and Sephadex LH-20 (40-70 $\mu \mathrm{m}$; Amersham Pharmacia Biotech AB). TLC was conducted on precoated silica gel plates GF254. HPLC separations were performed using an Agilent 1200 series pump equipped with a diode array detector, a semi-preparative Agilent Zorbax SB-C18 (5 $\mu \mathrm{m}$, $9.4 \times 250 \mathrm{~mm})$ column, and a semi-preparative Waters XBridge C-18 column $(5 \mu \mathrm{m}, 10 \times 250 \mathrm{~mm})$.

\section{Preparation of the acylated derivatives (1-5 and 10) of lycorine and lycorin-2-one}

A suspension of lycorine or lycorin-2-one (1 mmol), cinnamic acid or benzoic acid (1 eq.), dicyclohexylcarbodiimide (1 eq.), and 4-(N,N-dimethylamino)pyridine (1 eq.) in $25 \mathrm{~mL}$ of dry DMF was stirred for $12 \mathrm{~h}$ at room temperature. The urea byproduct was filtered, and the filtrate was evaporated. The resulting residue was purified by column chromatography on silica gel, using a mixture of hexane- $\mathrm{CHCl}_{3}-\mathrm{Me}_{2} \mathrm{CO}$ (10:2:1), $\mathrm{CHCl}_{3}$, and $\mathrm{CHCl}_{3}-\mathrm{MeOH}(100: 1)$ as eluent to yield the products (1-5 and 10).

\section{1,2-O,O'-Dibenzoyllycorine (1)}

Elution with hexane- $\mathrm{CHCl}_{3}-\mathrm{Me}_{2} \mathrm{CO}(10: 2: 1)$ and $\mathrm{CHCl}_{3}$ afforded 1[17] as a colorless solid, with a yield of $2.5 \%$; ${ }^{1} \mathrm{H}-\mathrm{NMR}\left(\mathrm{CDCl}_{3}, 400 \mathrm{MHz}\right): \delta 8.06(\mathrm{~d}, J=7.7 \mathrm{~Hz}, 2 \mathrm{H}$, H-2",6"), 7.91 (d, J = $\left.7.7 \mathrm{~Hz}, 2 \mathrm{H}, \mathrm{H}-2^{\prime}, 6^{\prime}\right), 7.55$ (m, 2H, H-4',4"), 7.42 (m, 4H, H-3',5',3",5"), 6.84 (s, 1H, H-8), 6.59 (s, 1H, H-12), 6.16 (br s, $1 \mathrm{H}, \mathrm{H}-1$ ), 5.90 and 5.86 (s, $1 \mathrm{H}$ each, $\mathrm{H}_{2}-11$ ), 5.69 (br s, $2 \mathrm{H}, \mathrm{H}-2,3$ ), 4.09 and 3.62 (br s, $1 \mathrm{H}$ each, $\mathrm{H}_{2}-7$ ), 3.47 and 2.53 (m, $1 \mathrm{H}$ each, $\mathrm{H}_{2}-5$ ), 3.19 (m, 1H, H-12c), 3.06 (m, 1H, H-12b), 2.77 (m, 2H, $\left.\mathrm{H}_{2}-4\right)$; ESIMS $m / z$ : $496[\mathrm{M}+\mathrm{H}]^{+}$; HREIMS for $\mathrm{C}_{30} \mathrm{H}_{25} \mathrm{NO}_{6}[\mathrm{M}]^{+}$: calcd. 495.1682; found: 495.1682.

\section{2-O-Benzoyllycorine (2)}

Elution with $\mathrm{CHCl}_{3}-\mathrm{MeOH}$ (100:1) afforded 2 [17] as a colorless solid, with a yield of $12.0 \%$; ${ }^{1} \mathrm{H}-\mathrm{NMR}\left(\mathrm{CDCl}_{3}\right.$, $400 \mathrm{MHz}):{ }^{1} \mathrm{H}-\mathrm{NMR}\left(\mathrm{CDCl}_{3}, 400 \mathrm{MHz}\right): \quad \delta 8.02$ (d, $\left.J=7.3 \mathrm{~Hz}, 2 \mathrm{H}, \mathrm{H}-2^{\prime}, 6^{\prime}\right), 7.54\left(\mathrm{t}, J=7.3 \mathrm{~Hz}, 1 \mathrm{H}, \mathrm{H}-4{ }^{\prime}\right)$, 7.40 (t, J= 7.3 Hz, 2H, H-3',5'), 6.80 (s, 1H, H-8), 6.58 (s, $1 \mathrm{H}, \mathrm{H}-12$ ), 5.87 and 5.87 (s, $1 \mathrm{H}$ each, $\mathrm{H}_{2}-11$ ), 5.58 (br s, $2 \mathrm{H}, \mathrm{H}-2,3$ ), 4.64 (br s, $1 \mathrm{H}, \mathrm{H}-1$ ), 4.16 and 3.55 (d, $J=$ $14.2 \mathrm{~Hz}, 1 \mathrm{H}$ each, $\left.\mathrm{H}_{2}-7\right), 3.37$ and 2.40 (m, $1 \mathrm{H}$ each, $\mathrm{H}_{2}-5$ ), 2.89 (d, $J=10.5 \mathrm{~Hz}, 1 \mathrm{H}, \mathrm{H}-12 \mathrm{~b}$ ), 2.83 (d, $J=10.5$ $\mathrm{Hz}, 1 \mathrm{H}, \mathrm{H}-12 \mathrm{c}), 2.67$ (m, 2H, $\left.\mathrm{H}_{2}-4\right) ;{ }^{13} \mathrm{C}-\mathrm{NMR}\left(\mathrm{CDCl}_{3}\right.$, $100 \mathrm{MHz}): \delta 166.0(\mathrm{O}(\mathrm{CO}) \mathrm{Ph}), 146.5(\mathrm{C}-10), 146.2 \times 2$

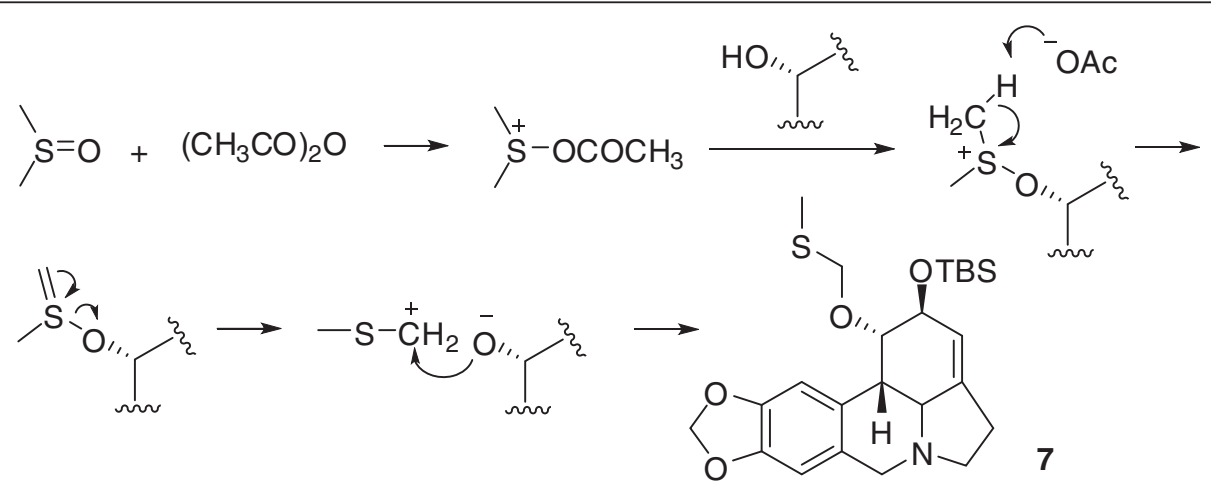

Scheme 1 The proposed mechanism for the formation of 7. 
(C-3a,9), 133.1 (C-4'), 130.0 and $129.8\left(\mathrm{C}-7 \mathrm{a}, 1^{\prime}\right), 129.7 \times$ 2 (C-2',6'), $128.3 \times 2$ (C-3',5'), 127.3 (C-12a), 113.7 (C-3), 107.5 (C-8), 104.8 (C-12), 100.9 (C-11), 74.1 (C-1), 69.0 (C-2), 60.7 (C-12c), 57.0 (C-7), 53.7 (C-5), 41.8 (C-12b), 28.7 (C-4); ESIMS $m / z$ : $392[\mathrm{M}+\mathrm{H}]^{+}$; HREIMS for $\mathrm{C}_{23} \mathrm{H}_{21} \mathrm{NO}_{5}[\mathrm{M}]^{+}$: calcd. 391.1420; found: 391.1413.

\section{1,2-O,O'-Di-trans-cinnamoyllycorine (3)}

Elution with hexane- $\mathrm{CHCl}_{3}-\mathrm{Me}_{2} \mathrm{CO}(10: 2: 1)$ and $\mathrm{CHCl}_{3}$ afforded 3 as a colorless solid, with a yield of $3.5 \%$; ${ }^{1} \mathrm{H}-\mathrm{NMR}\left(\mathrm{CDCl}_{3}, 400 \mathrm{MHz}\right): \delta 7.71(\mathrm{~d}, J=16.1 \mathrm{~Hz}$, $\left.1 \mathrm{H}, \mathrm{H}-7^{\prime \prime}\right), 7.64\left(\mathrm{~d}, J=16.1 \mathrm{~Hz}, 1 \mathrm{H}, \mathrm{H}-7^{\prime}\right), 7.52(\mathrm{~m}$, 2H, H-2",6"), 7.46 (m, 2H, H-2',6'), 7.38 (m, 3H, H$\left.3^{\prime \prime}, 4^{\prime \prime}, 5^{\prime \prime}\right), 7.34\left(\mathrm{~m}, 3 \mathrm{H}, \mathrm{H}-3^{\prime}, 4^{\prime}, 5^{\prime}\right), 6.84$ (s, 1H, H-8), 6.60 (s, 1H, H-12), 6.45 (d, $\left.J=16.1 \mathrm{~Hz}, 1 \mathrm{H}, \mathrm{H}-8^{\prime \prime}\right)$, $6.32\left(\mathrm{~d}, J=16.1 \mathrm{~Hz}, 1 \mathrm{H}, \mathrm{H}-8^{\prime}\right), 5.99$ (br s, $\left.1 \mathrm{H}, \mathrm{H}-1\right)$, 5.90 and 5.88 (s, $1 \mathrm{H}$ each, $\mathrm{H}_{2}-11$ ), 5.65 (br s, $1 \mathrm{H}$, $\mathrm{H}-2$ ), 5.51 (br s, $1 \mathrm{H}, \mathrm{H}-3), 4.18$ and $3.62(\mathrm{~d}, J=11.4$ $\mathrm{Hz}, 1 \mathrm{H}$ each, $\left.\mathrm{H}_{2}-7\right), 3.40$ and 2.50 (m, $1 \mathrm{H}$ each, $\left.\mathrm{H}_{2}-5\right), 3.06$ (d, $\left.J=9.6 \mathrm{~Hz}, 1 \mathrm{H}, \mathrm{H}-12 \mathrm{c}\right), 2.97(\mathrm{~m}, 1 \mathrm{H}$, $\mathrm{H}-12 \mathrm{~b}), 2.73\left(\mathrm{~m}, 2 \mathrm{H}, \mathrm{H}_{2}-4\right) ;{ }^{13} \mathrm{C}-\mathrm{NMR}\left(\mathrm{CDCl}_{3}, 100\right.$ $\mathrm{MHz}): \delta 165.8$ and 165.5 (C-9',9"), 146.4 (C-10), 145.8 and $145.5 \times 3$ (C-3a,9,7',7"), 134.3 and 134.1 (C-1',1"), 130.4 and 130.4 (C-7a,12a), $128.9 \times 2$ and $128.8 \times 2$ (C-3',5',3",5"), $128.1 \times 4$ (C-2',6',2",6"), $126.6 \times 2$ (C-4',4"), 117.7 and $117.3\left(\mathrm{C}-8^{\prime}, 8^{\prime \prime}\right), 114.3$ (C-3), 107.4 (C-8), 105.2 (C-12), 101.0 (C-11), 70.7 (C-1), 69.1 (C-2), 61.3 (C-12c), 56.7 (C-7), 53.7 (C-5), 40.4 (C-12b), 28.8 (C-4); ESIMS $m / z: 548[\mathrm{M}+\mathrm{H}]^{+}$; HREIMS for $\mathrm{C}_{34} \mathrm{H}_{29} \mathrm{NO}_{6}[\mathrm{M}]^{+}$: calcd. 547.1995; found: 547.1999 .

\section{2-O-trans-Cinnamoyllycorine (4)}

Elution with $\mathrm{CHCl}_{3}-\mathrm{MeOH}(100: 1)$ afforded 4 as a colorless solid, with a yield of $16.7 \%$; ${ }^{1} \mathrm{H}-\mathrm{NMR}\left(\mathrm{CDCl}_{3}, 400\right.$ $\mathrm{MHz}): \delta 7.70\left(\mathrm{~d}, J=16.1 \mathrm{~Hz}, 1 \mathrm{H}, \mathrm{H}-7^{\prime}\right), 7.50(\mathrm{~m}, 2 \mathrm{H}$, H-2',6'), 7.38 (m, 3H, H-3',4',5'), 6.84 (s, 1H, H-8), 6.61 (s, 1H, H-12), $6.43(\mathrm{~d}, J=16.1 \mathrm{~Hz}, 1 \mathrm{H}, \mathrm{H}-8$ '), 5.91 and 5.90 (s, $1 \mathrm{H}$ each, $\mathrm{H}_{2}-11$ ), 5.57 (br s, $1 \mathrm{H}, \mathrm{H}-2$ ), 5.48 (br s, $1 \mathrm{H}, \mathrm{H}-3$ ), 4.62 (br s, $1 \mathrm{H}, \mathrm{H}-1), 4.14$ and 3.68 (d, $J=14.1$ $\mathrm{Hz}, 1 \mathrm{H}$ each, $\left.\mathrm{H}_{2}-7\right), 3.38$ and 2.59 ( $\mathrm{m}, 1 \mathrm{H}$ each, $\mathrm{H}_{2}-5$ ), 3.01 (m, 1H, H-12b), 2.82 (d, $J=8.7 \mathrm{~Hz}, 1 \mathrm{H}, \mathrm{H}-12 \mathrm{c}$ ), $2.71\left(\mathrm{~m}, 2 \mathrm{H}, \mathrm{H}_{2}-4\right)$; ${ }^{13} \mathrm{C}-\mathrm{NMR}\left(\mathrm{CDCl}_{3}, 100 \mathrm{MHz}\right)$ : S 166.3 (C-9'), 146.4 (C-10), $145.5 \times 3$ (C-3a,9,7'), 134.2 $\left(\mathrm{C}-1^{\prime}\right), 130.4 \times 2(\mathrm{C}-7 \mathrm{a}, 12 \mathrm{a}), 128.9 \times 2\left(\mathrm{C}-3^{\prime}, 5^{\prime}\right), 128.1 \times 2$ (C-2',6'), 127.3 (C-4'), 117.7 (C-8'), 113.8 (C-3), 107.6 (C-8), 104.8 (C-12), 101.0 (C-11), 73.5 (C-1), 68.9 (C-2), 60.5 (C-12c), 56.5 (C-7), 53.8 (C-5), 41.3 (C-12b), 28.9 (C-4); ESIMS $m / z$ : $418[\mathrm{M}+\mathrm{H}]^{+}$; HREIMS for $\mathrm{C}_{25} \mathrm{H}_{23} \mathrm{NO}_{5}[\mathrm{M}]^{+}$: calcd. 417.1576; found: 417.1567.

\section{1-O-trans-Cinnamoyllycorine (5)}

Elution with $\mathrm{CHCl}_{3}-\mathrm{MeOH}(50: 1)$ afforded 5 as a colorless solid, with a yield of $1.4 \%$; ${ }^{1} \mathrm{H}-\mathrm{NMR}\left(\mathrm{CDCl}_{3}, 400\right.$
$\mathrm{MHz}):{ }^{1} \mathrm{H}-\mathrm{NMR}\left(\mathrm{CDCl}_{3}, 400 \mathrm{MHz}\right): \delta 7.59(\mathrm{~d}, J=16.0$ $\left.\mathrm{Hz}, 1 \mathrm{H}, \mathrm{H}-7^{\prime}\right), 7.45$ (m, 2H, H-2',6') 7.33 (m, 3H, H-3',4',5'), 6.64 (s, 1H, H-8), 6.59 (s, 1H, H-12), 6.28 (d, $\left.J=16.0 \mathrm{~Hz}, 1 \mathrm{H}, \mathrm{H}-8^{\prime}\right), 5.89$ and $5.87(\mathrm{~s}, 1 \mathrm{H}$ each, $\mathrm{H}_{2}-11$ ), 5.72 (br s, $1 \mathrm{H}, \mathrm{H}-1$ ), 5.59 (br s, $1 \mathrm{H}, \mathrm{H}-3$ ), 4.27 (br s, $1 \mathrm{H}, \mathrm{H}-2), 4.19$ and 3.61 (d, $J=14.0 \mathrm{~Hz}, 1 \mathrm{H}$ each, $\left.\mathrm{H}_{2}-7\right), 3.37$ and $2.51\left(\mathrm{~m}, 1 \mathrm{H}\right.$ each, $\left.\mathrm{H}_{2}-5\right), 2.95(\mathrm{~m}, 2 \mathrm{H}$, $\mathrm{H}-12 \mathrm{~b}, 12 \mathrm{c}), 2.67$ (m, 2H, H $\left.\mathrm{H}_{2}-4\right) ;{ }^{13} \mathrm{C}-\mathrm{NMR}\left(\mathrm{CDCl}_{3}, 100\right.$ $\mathrm{MHz}): \delta 166.6$ (C-9'), 146.6 (C-10), 146.3 and $145.5 \times 2$ (C-3a,9,7'), $134.1\left(\mathrm{C}-1^{\prime}\right), 130.4 \times 2$ (C-7a,12a), $128.8 \times 2$ (C-3',5'), $128.1 \times 2$ (C-2',6'), 127.1 (C-4'), $117.5\left(\mathrm{C}-8^{\prime}\right)$, 113.8 (C-3), 107.4 (C-8), 104.9 (C-12), 100.9 (C-11), 72.6 (C-1), 69.4 (C-2), 61.5 (C-12c), 56.8 (C-7), 53.7 (C-5), 39.1 (C-12b), 28.7 (C-4); ESIMS $m / z: 418[\mathrm{M}+\mathrm{H}]^{+}$; HREIMS for $\mathrm{C}_{25} \mathrm{H}_{23} \mathrm{NO}_{5}[\mathrm{M}]^{+}$: calcd. 417.1576; found: 417.1583 .

\section{1-O-trans-Cinnamoyllycorin-2-one (10)}

Elution with hexane- $\mathrm{CHCl}_{3}-\mathrm{Me}_{2} \mathrm{CO}$ (10:2:1), $\mathrm{CHCl}_{3}$, and $\mathrm{CHCl}_{3}-\mathrm{MeOH}(100: 1)$ afforded 10 as a colorless solid, with a yield of $62.7 \%$; ${ }^{1} \mathrm{H}-\mathrm{NMR}\left(\mathrm{CDCl}_{3}, 400 \mathrm{MHz}\right)$ : $\delta 7.63\left(\mathrm{~d}, J=16.0 \mathrm{~Hz}, 1 \mathrm{H}, \mathrm{H}-7^{\prime}\right), 7.44\left(\mathrm{~m}, 2 \mathrm{H}, \mathrm{H}-2^{\prime}, 6^{\prime}\right)$, 7.34 (m, 3H, H-3', $\left.4^{\prime}, 5^{\prime}\right), 6.79$ (s, 1H, H-8), 6.57 (s, 1H, H12), $6.28(\mathrm{~d}, J=16.0 \mathrm{~Hz}, 1 \mathrm{H}, \mathrm{H}-8 \mathrm{\prime}), 6.14(\mathrm{~d}, J=1.6 \mathrm{~Hz}$, $1 \mathrm{H}, \mathrm{H}-1$ ), 6.03 (br s, $1 \mathrm{H}, \mathrm{H}-3), 5.90$ and 5.89 (s, $1 \mathrm{H}$ each, $\mathrm{H}_{2}-11$ ), 4.18 and 3.65 (d, $J=14.0 \mathrm{~Hz}, 1 \mathrm{H}$ each, $\mathrm{H}_{2}-7$ ), 3.48 and $2.59\left(\mathrm{~m}, 1 \mathrm{H}\right.$ each, $\left.\mathrm{H}_{2}-5\right), 3.35$ (m, 2H, H12b,12c), 2.90 (m, 2H, H2 -4$)$; ${ }^{13} \mathrm{C}-\mathrm{NMR}\left(\mathrm{CDCl}_{3}, 100\right.$ $\mathrm{MHz}): \delta 204.4$ (C-2), 165.5 (C-9'), $146.7 \times 2$ (C-3a,10), $146.1 \times 2\left(\mathrm{C}-9,7^{\prime}\right), 134.1\left(\mathrm{C}-1^{\prime}\right), 130.5 \times 2(\mathrm{C}-7 \mathrm{a}, 12 \mathrm{a})$, $128.8 \times 2\left(\mathrm{C}^{\prime} 3^{\prime}, 5^{\prime}\right), 128.1 \times 2\left(\mathrm{C}-2^{\prime}, 6^{\prime \prime}\right), 125.2\left(\mathrm{C}-4{ }^{\prime}\right), 120.6$ (C-3), 116.9 (C-8'), 107.3 (C-8), 105.4 (C-12), 101.1 (C-11), 68.9 (C-1), 62.3 (C-12c), 56.1 (C-7), 53.2 (C-5), 45.4 (C-12b), 30.0 (C-4); ESIMS m/z: $416[\mathrm{M}+\mathrm{H}]^{+}$; HREIMS for $\mathrm{C}_{25} \mathrm{H}_{21} \mathrm{NO}_{5}[\mathrm{M}]^{+}$: calcd. 415.1420; found: 415.1418 .

\section{Synthesis of 1-O-acetyl-2-O-tert-butyldimethylsilyllycorine (6) and 2-0-tert-butyldimethylsilyl-1-0-(methylthio) methyllycorine (7)}

A solution of 2-O-tert-butyldimethylsilyllycorine [9] (60 $\mathrm{mg}, 0.150 \mathrm{mmol})$, dry dimethyl sulfoxide $(0.26 \mathrm{~mL})$, and acetic anhydride $(0.18 \mathrm{~mL})$ was stirred overnight at room temperature. Afterward, the reaction mixture was quenched with $\mathrm{H}_{2} \mathrm{O}(0.7 \mathrm{~mL})$ and aqueous $\mathrm{NH}_{4} \mathrm{OH}$ $(0.4 \mathrm{~mL})$. The resulting solution was extracted with $\mathrm{Et}_{2} \mathrm{O}$. The organic layer was separated, dried over $\mathrm{Na}_{2} \mathrm{SO}_{4}$, and then concentrated. The residue was purified by silica gel column chromatography (petrol/EtOAc, 10:1) and HPLC with a Waters XBridge C-18 column $(5 \mu \mathrm{m}$, $10 \times 250 \mathrm{~mm}$ ) using $\mathrm{MeOH}-\mathrm{H}_{2} \mathrm{O}(95: 5)$ as eluent to yield 6 [9] $\left(t_{\mathrm{R}}=9.876 \mathrm{~min}, 23 \mathrm{mg}, 0.0530 \mathrm{mmol}\right)$ and $7\left(t_{\mathrm{R}}=\right.$ $11.955 \mathrm{~min}, 17 \mathrm{mg}, 0.0368 \mathrm{mmol})$. 


\section{1-O-Acetyl-2-O-tert-butyldimethylsilyllycorine (6)}

A white solid, yield $35.4 \%$; ${ }^{1} \mathrm{H}-\mathrm{NMR}\left(\mathrm{CDCl}_{3}, 400 \mathrm{MHz}\right)$ : $\delta 6.73$ (s, 1H, H-8), 6.56 (s, 1H, H-12), 5.92 and 5.91 (s, $1 \mathrm{H}$ each, $\mathrm{H}_{2}-11$ ), 5.55 (br s, $1 \mathrm{H}, \mathrm{H}-1$ ), 5.39 (br s, $1 \mathrm{H}$, $\mathrm{H}-3$ ), 4.17 (br s, $1 \mathrm{H}, \mathrm{H}-2$ ), 4.14 and 3.52 (d, $J=14.1 \mathrm{~Hz}$, $1 \mathrm{H}$ each, $\left.\mathrm{H}_{2}-7\right), 3.36$ and 2.37 ( $\mathrm{m}, 1 \mathrm{H}$ each, $\left.\mathrm{H}_{2}-5\right), 2.94$ $(\mathrm{d}, J=8.8 \mathrm{~Hz}, 1 \mathrm{H}, \mathrm{H}-12 \mathrm{~b}), 2.74(\mathrm{~d}, J=8.8 \mathrm{~Hz}, 1 \mathrm{H}$, $\mathrm{H}-12 \mathrm{c}), 2.63\left(\mathrm{~m}, 2 \mathrm{H}, \mathrm{H}_{2}-4\right), 1.94$ (s, 3H, O(CO) $\left.\mathrm{CH}_{3}\right)$, 0.89 (s, 9H, $\left.\mathrm{SiC}\left(\mathrm{CH}_{3}\right)_{3}\right), 0.19$ and 0.11 (s, 3H each, Si $\left.\left(\mathrm{CH}_{3}\right)_{2}\right)$; ESIMS $m / z$ : $444[\mathrm{M}+\mathrm{H}]^{+} ;$HREIMS for $\mathrm{C}_{24} \mathrm{H}_{33} \mathrm{NO}_{5} \mathrm{Si}[\mathrm{M}]^{+}$: calcd. 443.2128; found: 443.2127.

\section{2-O-tert-Butyldimethylsilyl-1-O-(methylthio)methyllycorine (7)} A pale yellow solid, yield $24.5 \%$; ${ }^{1} \mathrm{H}-\mathrm{NMR}\left(\mathrm{CDCl}_{3}\right.$, $400 \mathrm{MHz}$ ): $\delta 7.02$ (s, 1H, H-8), 6.56 (s, 1H, H-12), 5.91 and 5.91 (s, $1 \mathrm{H}$ each, $\mathrm{H}_{2}-11$ ), 5.42 (br s, $1 \mathrm{H}$, $\mathrm{H}-3), 4.66$ and $4.62(\mathrm{~d}, J=12.0 \mathrm{~Hz}, 1 \mathrm{H}$ each, $\mathrm{OCH}_{2} \mathrm{SCH}_{3}$ ), 4.51 (br s, $\left.1 \mathrm{H}, \mathrm{H}-1\right), 4.34$ (br s, $1 \mathrm{H}$, $\mathrm{H}-2), 4.12$ and $3.50\left(\mathrm{~d}, J=14.0 \mathrm{~Hz}, 1 \mathrm{H}\right.$ each, $\mathrm{H}_{2}-7$ ), 3.34 and $2.33\left(\mathrm{~m}, 1 \mathrm{H}\right.$ each, $\left.\mathrm{H}_{2}-5\right), 2.88(\mathrm{~d}, J=10.4$ $\mathrm{Hz}, 1 \mathrm{H}, \mathrm{H}-12 \mathrm{~b}), 2.73$ (d, $J=10.4 \mathrm{~Hz}, 1 \mathrm{H}, \mathrm{H}-12 \mathrm{c}$ ), $2.61\left(\mathrm{~m}, 2 \mathrm{H}, \mathrm{H}_{2}-4\right), 1.97$ (s, 3H, $\left.\mathrm{SCH}_{3}\right), 0.89$ (s, 9H, $\left.\mathrm{SiC}\left(\mathrm{CH}_{3}\right)_{3}\right), 0.16$ and 0.12 (s, $3 \mathrm{H}$ each, $\left.\mathrm{Si}\left(\mathrm{CH}_{3}\right)_{2}\right)$; ESIMS $m / z$ : $462[\mathrm{M}+\mathrm{H}]^{+}$; HREIMS for $\mathrm{C}_{24} \mathrm{H}_{35} \mathrm{NO}_{4-}$ SiS $[\mathrm{M}]^{+}$: calcd. 461.2056; found: 461.2058 .

\section{Lycorin-2-one (8)}

Lycorine [10,12] (1 g, $3.481 \mathrm{mmol})$, PCC (6.657 g, $30.886 \mathrm{mmol})$ and silica gel $(6.657 \mathrm{~g})$ in anhydrous DMF $(50 \mathrm{~mL})$ were stirred at room temperature for $24 \mathrm{~h}$. Afterward, the reaction mixture was filtrated through a pad of Celite. The filtrate was then poured into water, adjusted the $\mathrm{pH}$ to 9 using ammonia, and extracted with $\mathrm{CHCl}_{3}$. The solvent was evaporated under reduced pressure. The residue was purified by silica gel column chromatography $\left(\mathrm{CHCl}_{3}-\mathrm{MeOH}, 20: 1\right)$ to yield $8[18,19](70$ $\mathrm{mg}, 0.245 \mathrm{mmol})$.

A gray powder, yield $7.0 \% ;{ }^{1} \mathrm{H}-\mathrm{NMR}\left(\mathrm{CDCl}_{3}, 500\right.$ $\mathrm{MHz}$ ): $\delta 6.77$ (s, 1H, H-8), 6.60 (s, 1H, H-12), 5.97 and 5.95 (s, $1 \mathrm{H}$ each, $\mathrm{H}_{2}-11$ ), 5.93 (br s, $\left.1 \mathrm{H}, \mathrm{H}-3\right), 4.55$ (d, $J=2.3 \mathrm{~Hz}, 1 \mathrm{H}, \mathrm{H}-1), 4.16$ and $3.64(\mathrm{~d}, J=14.0 \mathrm{~Hz}, 1 \mathrm{H}$ each, $\mathrm{H}_{2}-7$ ), 3.45 and 2.53 (m, $1 \mathrm{H}$ each, $\mathrm{H}_{2}-5$ ), 3.25 (br s, $1 \mathrm{H}, \mathrm{H}-12 \mathrm{~b}), 3.14$ (d, $J=9.4 \mathrm{~Hz}, 1 \mathrm{H}, \mathrm{H}-12 \mathrm{c}$ ), 2.86 (br s, $\left.2 \mathrm{H}, \mathrm{H}_{2}-4\right)$; ESIMS $m / z$ : $286\left[\mathrm{M}+\mathrm{H}^{+}\right.$; HREIMS for $\mathrm{C}_{16} \mathrm{H}_{15} \mathrm{NO}_{4}[\mathrm{M}]^{+}$: calcd. 285.1001; found: 285.1000.

\section{1-O-Acetyllycorin-2-one (9)}

A suspension of (20 mg, $0.0702 \mathrm{mmol})$ of lycorin-2-one (8) in $0.5 \mathrm{~mL}$ of pyridine and $0.5 \mathrm{~mL} \mathrm{Ac}_{2} \mathrm{O}$ was stirred for $12 \mathrm{~h}$ at room temperature and then $20 \mathrm{~mL}$ of water was added. The solution was adjusted to $\mathrm{pH} 9$ using ammonia $(5 \mathrm{~mL})$ and extracted with $\mathrm{CHCl}_{3}$ for four times before the removal of $\mathrm{CHCl}_{3}$. The resulting residue was purified by prep. TLC $\left(\mathrm{CHCl}_{3}-\mathrm{MeOH}, 30: 1\right)$ to yield 9 (5 mg, $0.0153 \mathrm{mmol}$ ).

A gray solid, yield $21.8 \%$; ${ }^{1} \mathrm{H}-\mathrm{NMR}\left(\mathrm{CDCl}_{3}, 400\right.$ $\mathrm{MHz}): \delta 6.72(\mathrm{~s}, 1 \mathrm{H}, \mathrm{H}-8), 6.57$ (s, 1H, H-12), 6.00 and $5.99\left(\mathrm{~s}, 1 \mathrm{H}\right.$ each, $\left.\mathrm{H}_{2}-11\right), 5.93$ and $5.92(\mathrm{br}, 1 \mathrm{H}$ each, $\mathrm{H}-1,3), 4.17$ and $3.61(\mathrm{~d}, J=14.1 \mathrm{~Hz}, 1 \mathrm{H}$ each, $\mathrm{H}_{2}-7$ ), 3.48 and 2.52 ( $\mathrm{m}, 1 \mathrm{H}$ each, $\mathrm{H}_{2}-5$ ), 3.26 (br d, $J=10.0 \mathrm{~Hz}, 1 \mathrm{H}, \mathrm{H}-12 \mathrm{~b}), 3.16$ (d, $J=10.0 \mathrm{~Hz}, 1 \mathrm{H}, \mathrm{H}-12 \mathrm{c})$, $2.86\left(\mathrm{~m}, 2 \mathrm{H}, \mathrm{H}_{2}-4\right), 1.96$ (s, 3H, O $\left.(\mathrm{CO}) \mathrm{CH}_{3}\right)$; ESIMS $m / z: 328[\mathrm{M}+\mathrm{H}]^{+}$; HREIMS for $\mathrm{C}_{18} \mathrm{H}_{17} \mathrm{NO}_{5}[\mathrm{M}]^{+}$: calcd. 327.1107; found: 327.1105. The NMR spectra of compounds 1-10 were also available as a PDF file (Additional file 1).

\section{Cholinesterase inhibitory activity}

AChE/BChE inhibitory activity of compounds 1-10 (purity $>95 \%$ ) was assayed using the spectrophotometric method developed by Ellman et al. [14], with slight modification. $S$-Acetylthiocholine iodide, $S$-butyrylthiocholine iodide, 5,5'-dithio-bis-(2-nitrobenzoic) acid (DTNB, Ellman's reagent), hAChE, and hBChE, were purchased from Sigma Chemical. The test compounds were dissolved in DMSO. The reaction mixture contained $110 \mu \mathrm{L}$ of phosphate buffer ( $\mathrm{pH} 8.0), 10 \mu \mathrm{L}$ of test compounds $(50 \mu \mathrm{M})$, and $40 \mu \mathrm{L}$ of hAChE or hBChE $(0.04 \mathrm{U} / 100 \mu \mathrm{L})$, and the mixture was incubated for $20 \mathrm{~min}\left(30^{\circ} \mathrm{C}\right)$. Subsequently, the reaction was initiated by the addition of $20 \mu \mathrm{L}$ of DTNB (6.25 $\mathrm{mM}$ ) and $20 \mu \mathrm{L}$ of $\mathrm{ACh}$ or butyrylthiocholine (BCh) for hAChE or hBChE inhibitory activity, respectively. Hydrolysis of ACh or BCh was monitored at $405 \mathrm{~nm}$ after $30 \mathrm{~min}$. All reactions were performed in triplicate. Inhibition percentage was calculated as follows: $\%$ inhibition $=(E-S) / E \times 100$, where $E$ is the enzyme activity without the test compounds and $S$ is the enzyme activity with the test compounds. Inhibition curves were obtained for each compound by plotting the inhibition percentage versus the logarithm of the inhibitor concentration in the assay solution. Linear regression parameters were determined for each curve, and the $\mathrm{IC}_{50}$ values were extrapolated. The same procedure was applied for the positive control tacrine (Sigma, purity 98\%) and galanthamine (purity >95\%) [12]. The study was approved by the ethical committee in Kunming Institute of Botany (reference number 1205) and performed according to the Helsinki Declaration.

\section{Conclusion}

A series of lycorine derivatives (1-10) were synthesized and evaluated for anti-cholinesterase activity. The novel compound 2-O-tert-butyldimethylsilyl-1-O-(methylthio) methyllycorine (7) was a dual hAChE and hBChE inhibitor. The structure-activity relationships indicated that (i) 
the 1-O-(methylthio)methyl substituent in lycorine is better than the 1-O-acetyl group for the inhibition of cholinesterase; (ii) the acylated or etherified derivatives of lycorine and lycorin-2-one are more potent against $\mathrm{hBChE}$ than hAChE; and (iii) the oxidation of lycorine at $\mathrm{C}-2$ decreases the activity. Hence, further study on the modification of lycorine for the inhibition of $\mathrm{ChE}$ is necessary.

\section{Additional file}

Additional file 1: NMR spectra of compounds 1-10.

\section{Abbreviations}

$\mathrm{A} \beta$ : $\beta$-amyloid; Ach: Acetylcholine; AChE: Acetylcholinesterase; $\mathrm{AC}_{2} \mathrm{O}$ : Acetic anhydride; AD: Alzheimer's disease; BChE: Butyrylcholinesterase; ChE: Cholinesterase; DMAP: 4-(N,N-dimethylamino)pyridine; DMF: N,Ndimethylformamide; DTNB: 5,5'-dithio-bis-(2-nitrobenzoic) acid; DMSO: Dimethylsulphoxide; DCC: Dicyclohexylcarbodiimide; eeAChE: Electric eel acetylcholinesterase; ESIMS: Electrospray ionization mass spectrometry; EtOAc: Ethyl acetate; hAChE: Human acetylcholinesterase; hBChE: Human butyrylcholinesterase; HPLC: High pressure liquid chromatography; HREIMS: High resolution electrospray ionization mass spectrometry; $\mathrm{IC}_{50}$ : Concentration producing 50\% inhibition; $\mathrm{Me}_{2} \mathrm{CO}$ : Acetone; NMR: Nuclear magnetic resonance; PCC: Pyridinium chlorochromate; TBS: Tertbutyldimethylsilyl; TLC: Thin layer chromatography; TMS: Tetramethylsilane.

\section{Competing interests}

The authors declare that they have no competing interests.

\section{Authors' contributions}

YHW and CLL directed the whole study of the paper. The synthetic experiments were carried out by YHW and CDG. The bioassay was performed by QLW and HRL. YHW drafted the manuscript and CLL revised it. All authors read and approved the final manuscript.

\section{Acknowledgements}

This work was funded by the National Natural Science Foundation of China (Nos. 20972166, 31161140345, 31070288), the Natural Science Foundation of Yunnan Province, China (No. 2011FZ205), and the Ministry of Education of China through its 111 and 985 projects (Nos. B08044, MUC985-9, MUC9850601000101)

\section{Author details}

${ }^{1}$ Key Laboratory of Economic Plants and Biotechnology, Kunming Institute of Botany, Chinese Academy of Sciences, Kunming 650201, PR China. ${ }^{2}$ State Key Laboratory of Phytochemistry and Plant Resources in West China, Kunming Institute of Botany, Chinese Academy of Sciences, Kunming 650201, PR China. ${ }^{3}$ School of Chemistry and Chemical Engineering, Nanjing University, Nanjing 210093, PR China. ${ }^{4}$ College of Life and Environmental Sciences, Minzu University of China, Beijing 100081, PR China.

Received: 27 May 2012 Accepted: 24 August 2012

Published: 8 September 2012

\section{References}

1. Cisse M, Mucke L: Alzheimer's disease: A prion protein connection. Nature 2009, 457:1090-1091.

2. Leifer BP: Early diagnosis of Alzheimer's disease: clinical and economic benefits. J Am Geriatr Soc 2003, 51:S281-S288.

3. Mohamed T, Rao PPN: Alzheimers disease: emerging trends in small molecule therapies. Curr Med Chem 2011, 18:4299-4320.

4. Walsh R, Rockwood K, Martin E, Darvesh S: Synergistic inhibition of butyrylcholinesterase by galantamine and citalopram. Biochim Biophys Acta 2011, 1810:1230-1235.

5. Greig NH, Utsuki T, Ingram DK, Wang Y, Pepeu G, Scali C, Yu QS, Mamczarz J, Holloway HW, Giordano T: Selective butyrylcholinesterase inhibition elevates brain acetylcholine, augments learning and lowers Alzheimer $\beta$ amyloid peptide in rodent. Proc Nat Acad Sci USA 2005, 102:17213-17218.

6. Furukawa-Hibi Y, Alkam T, Nitta A, Matsuyama A, Mizoguchi H, Suzuki K, Moussaoui S, Yu QS, Greig NH, Nagai T: Butyrylcholinesterase inhibitors ameliorate cognitive dysfunction induced by amyloid-[beta] peptide in mice. Behav Brain Res 2011, 1:222-229.

7. Darvesh S, Cash MK, Reid GA, Martin E, Mitnitski A, Geula C: Butyrylcholinesterase is associated with $\beta$-amyloid plaques in the

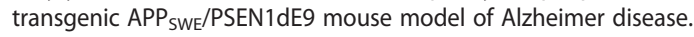
J Neuropathol Exp Neurol 2012, 71:2-14.

8. Elgorashi EE, Stafford Gl, van Staden J: Acetylcholinesterase enzyme inhibitory effects of Amaryllidaceae alkaloids. Planta Med 2004, 70: 260-262.

9. McNulty J, Nair JJ, Little JRL, Brennan JD, Bastida J: Structure-activity studies on acetylcholinesterase inhibition in the lycorine series of Amaryllidaceae alkaloids. Bioorg Med Chem Lett 2010, 20:5290-5294.

10. Wang YH, Zhang ZK, Yang FM, Sun QY, He HP, Di YT, Mu SZ, Lu Y, Chang Y, Zheng QT, Ding M, Dong JH, Hao XJ: Benzylphenethylamine alkaloids from Hosta plantaginea with inhibitory activity against tobacco mosaic virus and acetylcholinesterase. J Nat Prod 2007, 70:1458-1461.

11. Wang YH, Gao S, Yang FM, Sun QY, Wang JS, Liu HY, Li CS, Di YT, Li SL, He HP, Hao XJ: Structure elucidation and biomimetic synthesis of hostasinine A, a new benzylphenethylamine alkaloid from Hosta plantaginea. Org Lett 2007, 9:5279-5281.

12. Wang $H$, Wang $Y H$, Zhao FW, Huang $Q Q$, Xu JJ, Ma LJ, Long $C L$ : Benzylphenethylamine alkaloids from the bulbs and flowers of Lycoris radiata. Chin Herb Med 2011, 3:60-63.

13. Elgorashi E, Malan S, Stafford G, Van Staden J: Quantitative structureactivity relationship studies on acetylcholinesterase enzyme inhibitory effects of Amaryllidaceae alkaloids. S Afr J Bot 2006, 72:224-231.

14. Ellman GL, Courtney KD, Andres V, Featherstone RM: A new and rapid colorimetric determination of acetylcholinesterase activity. Biochem Pharmacol 1961, 7:88-95.

15. Christensen SM, Hansen HF, Koch T: Molar-scale synthesis of 1, 2: 5, 6-diO-isopropylidene-a-D-allofuranose: DMSO oxidation of 1, 2: 5, 6-di-O-


reduction. Org Process Res Dev 2004, 8:777-780.

16. Cavalli A, Bolognesi ML, Minarini A, Rosini M, Tumiatti $V$, Recanatini M, Melchiorre C: Multi-target-directed ligands to combat neurodegenerative diseases. J Med Chem 2008, 51:347-372

17. Evdokimov NM, Lamoral-Theys D, Mathieu V, Andolfi A, Frolova LV, Pelly SC, van Otterlo WAL, Magedov IV, Kiss R, Evidente A, Kornienko A: In search of a cytostatic agent derived from the alkaloid lycorine: Synthesis and growth inhibitory properties of lycorine derivatives. Bioorg Med Chem 2011, 19:7252-7261.

18. Lamoral-Theys D, Andolfi A, Van Goietsenoven G, Cimmino A, Le Calvé B, Wauthoz N, Mégalizzi V, Gras T, Bruyére C, Dubois J, Mathieu V, Kornienko A, Kiss R, Evidente A: Lycorine, the main phenanthridine Amaryllidaceae alkaloid, exhibits significant antitumor activity in cancer cells that display resistance to proapoptotic stimuli: an investigation of structure-activity relationship and mechanistic insight. J Med Chem 2009, 52:6244-6256.

19. Cedron JC, Gutierrez D, Flores N, Ravelo AG, Estevez-Braun A: Synthesis and antiplasmodial activity of lycorine derivatives. Bioorg Med Chem 2010, 18:4694-4701

doi:10.1186/1752-153X-6-96

Cite this article as: Wang et al:: Synthesis and biological evaluation of lycorine derivatives as dual inhibitors of human acetylcholinesterase and butyrylcholinesterase. Chemistry Central Journal 2012 6:96. 